\title{
Advanced ICT for Access of Visual Impaired People to Computers, Knowledge, Education and Culture
}

\author{
Dimitar Karastoyanov \\ Institute of Information and \\ Communication Technologies \\ Sofia, Bulgaria \\ dimikara@abv.bg
}

\author{
Stanislav Gyoshev \\ Institute of Information and \\ Communication Technologies \\ Sofia, Bulgaria \\ stanislavgyoshev@mail.bg
}

\author{
Ava Chikurteva \\ Institute of Information and \\ Communication Technologies \\ Sofia, Bulgaria \\ hawa_@abv.bg
}

Received: March 27, 2020. Revised: August 5, 2020. 2nd Revised: September 18, 2020.

Accepted: September 24, 2020. Published: September 29, 2020.

\begin{abstract}
The paper presents methods and tools for tactile access of people with visual impairments to a graphical computer interface, cultural and historical heritage and temperature in the home. Different size of graphical Braille screens on the base of linear electromagnetic micro drives, tactile graphical tiles and thermometer for visual impaired are described. The presented devices give access of visual impaired people to graphical computer interface (Windows), objects of cultural and historical heritage, as well as give possibilities for better education and perception of knowledge.
\end{abstract}

Keywords - graphical Braille screen, linear micro drive, tactile graphical tile, tactile thermometer

\section{INTRODUCTION}

Just a few decades ago, screens had only terminals and small desktops. Communication was via keyboards and text operating systems. Special buttons were developed for the visually impaired and Braille symbols were used. Modern computers, tablets and smartphones use graphical operating systems, mouses and touch screen. People with visual impairments do not have access through graphical communication and therefore do not have access to computers, education, scientific knowledge, and cultural and historical heritage. Here we present the methods and tools we have developed to provide this access for visually impaired people (with reduced sight or blind), [1], [2].

\section{GRAPHICAL BRAILLE SCREEN}

The team have a prototype of a Braille Screen/Display (Fig. 1), based on the 5 Bulgarian patents. Here we present the research on design, optimization and development of magnetic based linear actuator (Fig. 2), in order to show advantages of this devices, [3] - [7], [8].

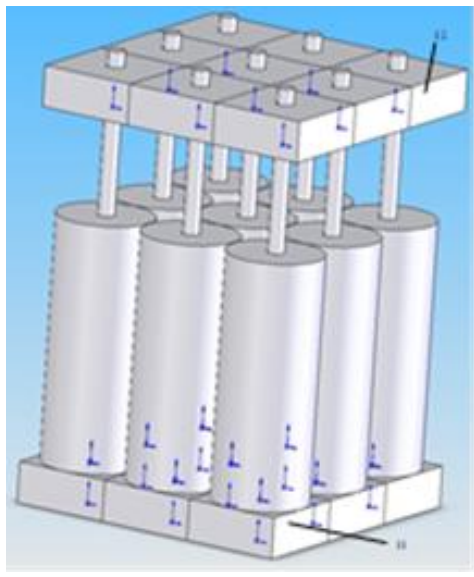

Fig. 1. Braille screen matrix.

\section{A. Braille Screen Design}

The Braille Display represents a matrix comprised of a base with fixed electromagnets. They are arranged thereon, including an outer cylindrical magnetic core, in which a winding magnetic core locking up the cylindrical magnetic core at the top side. A winding magnetic core locking up the cylindrical magnetic core at the bottom side are placed. The magnetic cores are with axial holes. Into the space between the windings is placed a movable non-magnetic cylindrical body, carrying an axially magnetized permanent cylindrical magnet and a non-magnetic needle. The needle is passing axially through the permanent magnet and the axial holes of the magnetic cores. On the top side of the permanent magnet is arranged a ferromagnetic disc having an axial hole. On its underside is arranged a ferromagnetic disc having an axial hole. The upper disc and the upper magnetic core have cylindrical poles and the lower magnetic core and the lower disc have conical poles. A lattice is placed above the electromagnets. The needles pass through the openings.

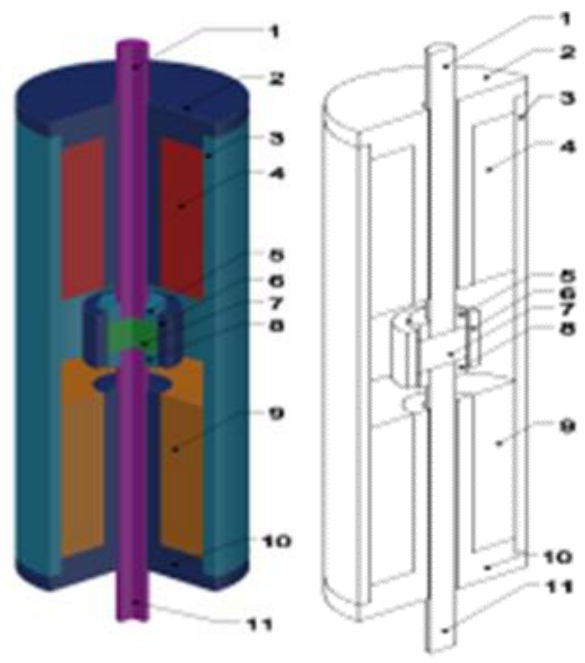

Fig. 2. Linear electromagnetic actuator

Principal geometry of the linear electromagnetic actuator with permanent magnet: 1 - Needle (shaft); 2 - Upper core; 3 - Outer core; 4 - Upper coil; 5 - Upper ferromagnetic disc; 6 Non-magnetic bush; 7 - Permanent magnet; 8 - Lower ferromagnetic disc; 9 - Lower coil; 10 - Lower core; 11 Needle (shaft).

\section{B. Actuator Construction}

The actuator is a linear electromagnetic micro drive. The mover is a permanent magnet. Its magnetization direction is along the axis of rotational symmetry. The upper and lower 
coil are connected in series. This connection is realized so that the flux created by each of them is in opposite directions in the mover zone. Thus by choosing proper power supply polarity, the motion of the mover will be in desired direction. For example, in order to have motion of the mover in upper direction, the upper coil has to be supplied in a way to create air gap magnetic flux, which is in the same direction as the one of the flux created by the permanent magnet. The lower coil in this case will create magnetic flux which is in opposite direction to the one of the magnetic flux created by the permanent magnet. In this case motion up will be observed. In order to have motion down, the lower coil should be supplied in a way so that its flux is in the same direction as the flux by the permanent magnet. The upper coil then will create magnetic flux in opposite direction. In order to fix the moving part to the Braille dot, non-magnetic shaft is used. Additional construction variants of the actuator have also been considered, in which two small ferromagnetic discs are placed on both sides - upper and lower - of the moving permanent magnet. This actuator is also energy efficient, as energy is used only for changing the position of the moving part from lower to upper and vice versa. No energy is used both at lower and at upper position. At these positions, the mover is kept fixed due to the force ensured by the permanent magnet (sticks to the core).

\section{Research and Optimization}

Static Force Characteristics are obtained for different construction parameters of the actuator. Magnetic field of the construction variant of the linear actuator with two ferromagnetic discs on both sides of the permanent magnet is analyzed with the help of the finite element method, [9], [10]. The optimality criterion for optimization is the minimal magneto motive force of the coils. The optimization factors are geometrical parameters (height of the permanent magnet, height of the ferromagnetic discs and height of the coils). The optimization is carried out subjecting to the following constraints - minimal electromagnetic force acting on the mover, minimal starting force and overall outer diameter of the actuator have been set. Minimization of magneto motive force is in direct correspondence to minimization of the energy consumption, [11].

\section{Use of the Braille Screen}

The graphical Braille display can be used as tactile communication interface for visual impaired people.

We consider the development of three variants of the Braille Display for visually impaired:

- Small, up to $24 \times 16$ pins. It will be used by children in schools for exploring simple graphics;

- Average, up to $48 \times 32$ pins. It will be used by visually impaired users when working with computers and graphical interface - Windows icons. In this case the Display will be able to show schedules with average complexity of surveys, statistics, and scientific articles.

- Large, up to $128 \times 96$ pins and more. These displays will be designed for museums, galleries, etc. They will present works of cultural and historical heritage - paintings, tapestries, icons, etc. The presentation will be accompanied by explanations of the context, possibilities for detailing, expansion and reduction of parts, etc.

\section{GRAPICAL TACTILE TILES}

In 2015 the team took part in the Exhibition "Pavia. The Battle The Future 1525-2015. Nothing is the same again", a satellite event of EXPO 2015 Milan, Italy, [2]. Seven famous medieval tapestries were shown as tactile plates to general public including visually impaired who could touch them Fig. 3. All tactile plates have a strip with title and key in Braille so that each image segment, marked with Braille symbols, can be read and interpreted. The visitors could move their fingers back and forth, trace the contours corresponding to a Braille key, and explore the plates with whole hand.

The next invention relates to a Tactile graphical tile for visually impaired, which will find application for the presentation of objects of cultural and historical heritage for users with impaired vision, who by touch can "see" and imagine the corresponding picture, icon, photo, tapestry and more, [12], [13], [14] - fig. 4a.

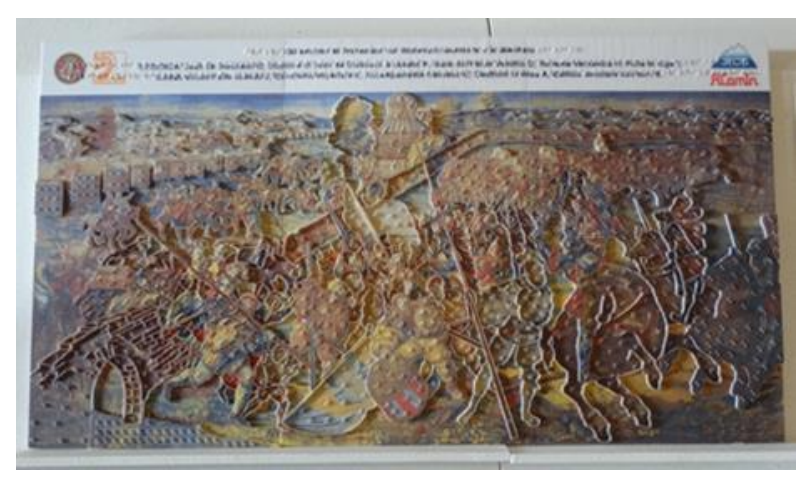

Fig. 3. Tactile tile on the base of a tapestry

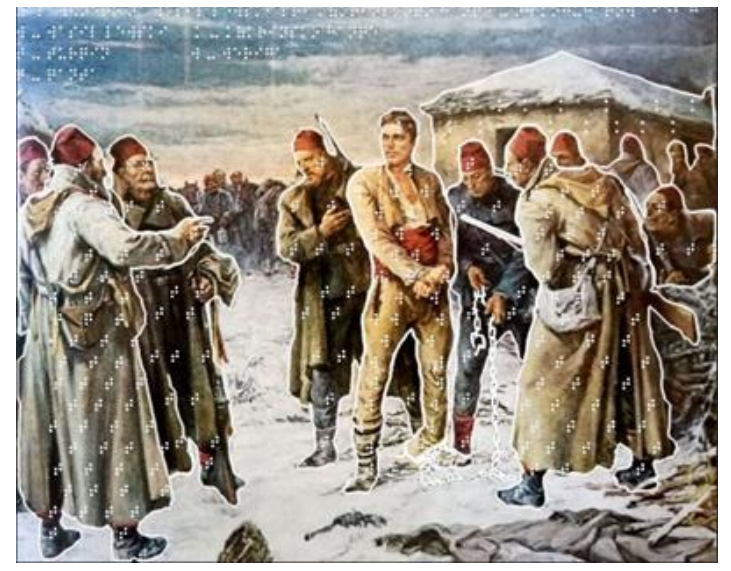

a) The origin painting "Capturing Vasil Levski"

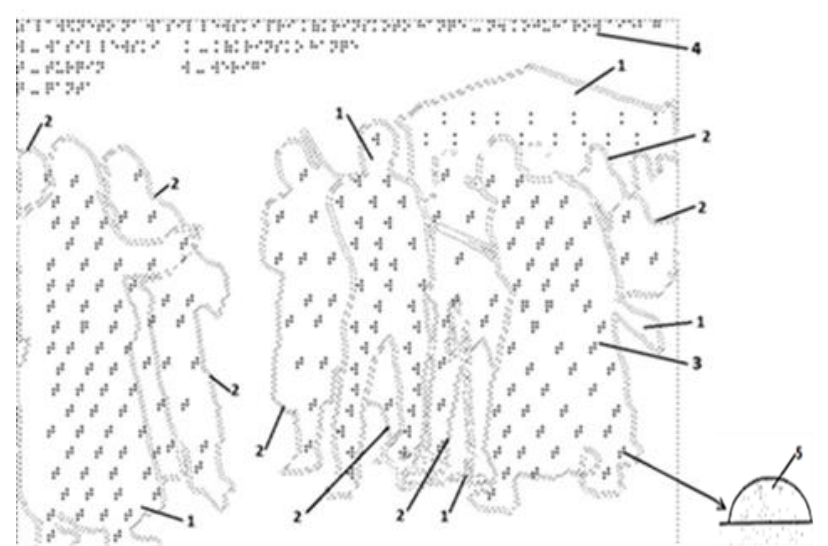


b) Tactile tile elements

Fig. 4. Tactile graphical tile

The tactile graphical tile for visually impaired (fig. 4. b) consists of figures (1), contours (2), Braille symbols (3) and Braille annotation (4), composed of hemispherically outwardly protruding buds (5), wherein the figures (1) are arranged in two levels on the tile. The low level is the surface of the tile and the high level is at a distance from the surface of the tile. The high level figures (1) being dense over the entire area of the corresponding figure (1) and the low level figures being outlined with contours (2). The height of the contours (2) being equal to the high level. Two adjacent figures (1) on the tile are of different levels, alternating "high-density solid figure (1)" with "low-level figure (1) outlined with contours (2)" and also inside the area of each figure (1) a Braille symbol (3) is placed, representing the first letter of the name of the figure (1). At one end of the tile a complete Braille annotation (4) is placed on the semantic context of the graphic images shown on the tile. The height of Braille symbols (3) representing the protruding buds (5) is equal to the height of the high level and the contours (2).

The advantage of the tactile graphical tile for visually impaired is that it can represent, in addition to symbols (letters and numbers), also graphic information - figures and images. Another advantage is that it can present any static flat object of cultural and historical heritage - a painting, an icon, a photo, a tapestry, etc., so that it can be "seen" and perceived by a visually impaired user by touching the figures with fingers.

We also plan to use a drone to create 3D models of large cultural and historical objects - monuments, buildings and more. Printed small 3D models of objects can serve to present the originals, including if the objects are expensive and unique or as educational content, be touched or given as souvenirs to children - Fig. 5 .

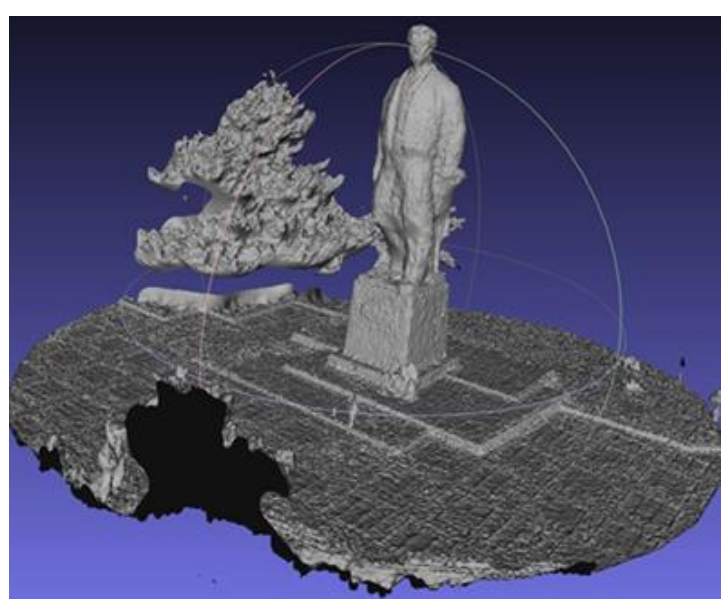

Fig. 5. The $10 \mathrm{~m}$ monument of Vasil Levski

\section{TACTILE THERMOMETER}

The invention THERMOMETER is designed to serve visually impaired people and will find application in industrial and household appliances such as boilers, irons, hotplates, kettles and more. The thermometer (Fig. 6) includes a hub 1 and a spiral bimetal plate 2 . The inner end 3 of the spiral bimetal plate 2 is mounted to the hub 1 and the outer end 4 of the spiral bimetal plate 2 is free, with the tip 5 of the outer end 4 being tapered. Above the outer end 4 of the spiral bimetallic plate 2 are tubular beds 6 with springs 7 and movable elements in the beds 6 - buttons 8 of different lengths. The bases 9 of the buttons 8 are tapered and the tips 10 of the buttons 8 are rounded. Under the influence of temperature, the helical bimetallic plate 2 extends radially and the outer end 4 moves in a circle, successively raise the buttons 8 depending on the temperature. As the temperature rises further, the spiral bimetal plate 2 raises several buttons 8 in succession. If the temperature starts to decrease, the spiral bimetal plate 2 shrinks backwards and releases several buttons 8 in succession, which are retracted by the action of the springs 7 in their beds 6 . The advantage of the thermometer for visually impaired people is its small mass and size, which makes it suitable for installation in domestic heating appliances - irons, hot plates, kettles, water heaters. Another advantage of the thermometer is that visually impaired people can be informed of the temperature by touching the buttons raised on the bimetal plate. A significant advantage of the thermometer is that it gives information about the temperature reached by how many buttons are held up, which cannot be detected by a visually impaired person through only one ON/OFF button raised, [15].

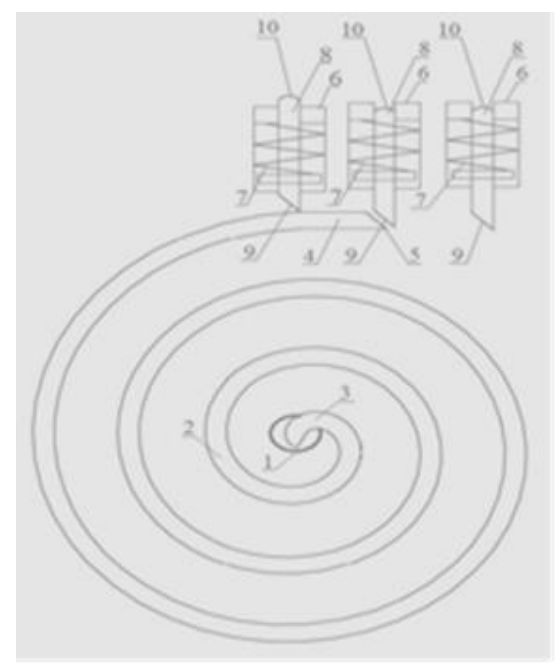

Fig. 6. The tactile thermometer

\section{3D TACTILE PERSEPTION}

Depending on the size and resolution (small, medium or large), the Braille screen can be used by children for simple charts, such as a graphical computer interface, in museums and galleries for the presentation of cultural and historical objects. In the large case, there may also be a dynamic representation of individual parts with different degrees of detail. The same goes for tactile graphic tiles. There is an opportunity to present a tactile tile through a large Braille screen if it has thousands of 'pixels'.

Tactile perception of environmental elements can be offered not only for users with visual impairments, not only in museums and at home.

3D models of historic monuments, famous buildings, human organs, etc., as well as small 3D tiles of paintings, icons, tapestries, etc., can be made cheaply from plastic a few centimeters in size and available to scholars and students (not only low sighted) in the lessons of the respective disciplines. At the end of the lesson, scholars and students can bring items and tiles home as souvenirs. This will greatly 
increase the interest and perception of the relevant knowledge by the children and young people.

\section{CONCLUSION}

People with visual impairments are a large group of citizens who have so far been denied access based on visual communication. Voice assistants are used as a means of perception. Higher tactile sensitivity is used through Braille books. So far, dynamic tactile perception has only been available via Braille terminals for Braille symbols - letters and numbers. Here we present three innovations - screen, tile and thermometer, helping visual impaired people have access to computers, knowledge, education and culture.

\section{ACKNOWLEDGMENT}

The paper is supported by the BG NSF Grant No DN 17/21-2017.

\section{REFERENCES}

[1] Karastoyanov, D., Control of robots and other mechatronic systems, 2010, Academy Publishing House, Sofia, Bulgaria, (in Bulgarian), ISBN 987-954-322-415-9

[2] Kantoni V., Karastoyanov D., Mosconi M., Setti A., CVML and SMART LAB at the Exibition (Pavia. The Battle. The Future - 15252015, Nothing was the same again)., Pavia University Press, 2016, Pavia, Italy, (in English and Italian), ISBN: 978-88-6952-035-8 (book), ISBN: 978-88-6952-036-5 (e-book)

[3] Karastoyanov D., Braille screen, Bulgarian Patent No 66520, (2016).

[4] Karastoyanov D., Simeonov S., Braille display, Bulgarian Patent No 66527, (2016)

[5] Karastoyanov D., Yatchev I., Hinov K., Rachev T., Braille screen, Bulgarian Patent No 66562, (2017)

[6] Karastoyanov D., Yatchev Y., Hinov K., Rachev P., Braille Screen, Bulgarian Patent No 66702, (2018)
[7] Karastoyanov D., Yatchev Y., Hinov K., Balabozov I., Braille Display,Bulgarian Patent No 66734, (2018)

[8] Gyoshev, S., Karastoyanov, D., Stoimenov, N., Cantoni, V., Lombardi, L., Setti, A. Exploiting a graphical braille display for art masterpieces (2018) Lecture Notes in Computer Science (including subseries Lecture Notes in Artificial Intelligence and Lecture Notes in Bioinformatics), 10897 LNCS, pp. 237-245. DOI: 10.1007/978-3319-94274-2_33,

[9] Karastoyanov D., and V1. Kotev. Electromagnetic Microdrives for Braille Screen: Control and Circuit Testing, Intern. Journal of Materials Science and Engineering, vol. 3, (1), March 2015, pp: 1-6, ISSN 2315-4527, doi: 10.12720/ijmse.3.1.1-6.

[10] Karastoyanov D., L. Doukovska, V. Atanassova, Electromagnetic Linear Micro Drives for Braille Screen: Characteristics, Control and Optimization, Proc. of the Third International Conference on Telecommunications and Remote Sensing - ICTRS'14, 26-27 June 2014, Luxembourg, Grand Duchy of Luxembourg, ISBN 978-989758-033-8, pp. 88-93, 2014.

[11] Karastoyanov, D. Hybrid electromagnetic systems for energy efficiency of electrical systems (2018) AIP Conference Proceedings, 2022, art. no. 020016, DOI: 10.1063/1.5060696,

https://www2.scopus.com/inward/record.uri?eid=2-s2.0-

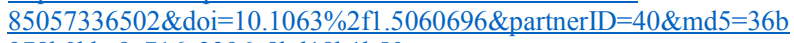
978b0bbc9c716c3396a8bd18b1b50

[12] Gyoshev S., Popov B., Karastanev St.. ICT for 3D modeling and 3D tactile visualization of sites of cultural and historical heritage. Conference proceedings, 70, Croatian society for mechanical technologies, Croatia, 2018, ISSN:1847-7917, 53-56

[13] Karastoyanov D., Stoimenov N., Gyoshev S., Tactile graphical tile for visual impaired people., Bulgarian patent application No 112716, (2018)

[14] Cantoni, V., Lombardi, L., Setti, A., Gyoshev, S., Karastoyanov, D., Stoimenov, N. Art masterpieces accessibility for blind and visually impaired people (2018) Lecture Notes in Computer Science (including subseries Lecture Notes in Artificial Intelligence and Lecture Notes in Bioinformatics), 10897 LNCS, pp. 267-274. DOI: 10.1007/978-3-319-94274-2_37

[15] Karastoyanov D., Stoimenov N, Gyoshev S, Thermometer, Bulgarian patent application No 11368, (2016)

\section{Creative Commons Attribution License 4.0} (Attribution 4.0 International, CC BY 4.0)

This article is published under the terms of the Creative Commons Attribution License 4.0

https://creativecommons.org/licenses/by/4.0/deed.en_US 